\title{
IAMJ
}

INTERNATIONAL AYURVEDIC MEDICAL JOURNAL

\section{A CASE STUDY OF ALCOHOLIC PATIENT}

\author{
Noori Aara ${ }^{1}$, Parmanand Upadhyay ${ }^{2}$, Sunita Godara ${ }^{3}$, Mohd. Adil Ansari ${ }^{4}$
}

${ }^{1}$ MD scholar, P.G. Dept, Agad Tantra Evam Vyavahar Ayurveda, Dsrrau, Jodhpur, Rajasthan, India

${ }^{2}$ Associate Professor \& HOD, P.G. Dept, Agad Tantra Evam Vyavahar Ayurveda, Dsrrau, Jodhpur, Rajasthan, India

${ }^{3}$ Assistant Professor, P.G. Dept, Agad Tantra Evam Vyavahar Ayurveda, Dsrrau, Jodhpur, Rajasthan India

${ }^{4}$ MD scholar, P.G. Dept, Kayachikitsa, Dsrrau, Jodhpur, Rajasthan, India

\section{Corresponding Author: nooriaara123@gmail.com}

\section{https://doi.org/10.46607/iamj3908122020}

(Published online: December 2020)

Open Access

(C) International Ayurvedic Medical Journal, India 2020

Article Received: 26/11/2020 - Peer Reviewed: 28/11/2020 - Accepted for Publication: 02/12/2020

Check for updates

\begin{abstract}
In this Present era of globalization, the stress of highly competitive work environment, increasing demands of family and friends on their time, fast food culture, fast lifestyle etc. are pushing executives, employees and even common men in to pressure cooker like existence. people found their all solutions by various addiction and one of which very common is that of alcohol. which gives pleasures and relaxation to millions but later it developed into frequent use of alcohol. Alcohol is strong associated with a wide range of mental health problems. In view of Ayurveda Madya (ethyl alcohol) is poison. though the Madya is poison, when person drink it in a adiequate dose, by proper manner with proper diet, it like as Amrit. But if we took a drink in over dose and by improper manner then it's destroyed the Oja dhatu in human body. A 47 years old male chronic and heavy alcohol drinker for 10 years. Consequently, he started having sweating, nausea, vomiting, tremors, loss of appetite, constipation, disturbed sleep, slurred speech, headache, constipation as alcohol withdrawal symptoms. The patient was treated with Nashamukti Yog-1 (Kalpityog), Ashavgandha Churna, Shatavari churna, Chopchini churna, Madhuyashti churna, Shiva gutika, Panchsakar churna, Shirodhara with Mahanarayan Tail. The treatment approaches to improve in condition of patient with improvement in sign and symptoms of alcohol withdrawal. The result assessment is done on the basis of the Clinical Institute Withdrawal Assessment of Alcohol revised scale (CIW-Ar score).
\end{abstract}

Keywords: Alcohol dependence, CIW-Ar score 


\section{INTRODUCTION}

That which produces Mada is called Madya ${ }^{1}$, the disease produced due to improper use of Madya is called Madatyaya ${ }^{2}$. Madya is explained to have qualities or gunas similar to visha, but in a milder form and also opposite to qualities of ojas ${ }^{3}$. The alteration of ojas results in the imbalance between the dhatus and also in immune-compromise in the individual. so, if it is not administered properly, results in harm to the body and is termed as slow poison, by many ${ }^{4}$.

Madatyaya is produced when person takes the Madya without considering Prakriti, Satmya, Agni etc ${ }^{5}$. Madatyaya is a Tridoshaja Vyadhi mainly Kapha Sthan is vitiated along with $A g n i^{6}$. when the chronic alcoholic addicted patient stop to drink, sudden withdrawal causes group of clinical manifestation called as alchohol withdrawal syndrome. Acharya Kashap also described this term under the heading of Panatyaya(Madatyaya), means sudden stop of alcohol leads to symptoms. The chronic drinker who has not get Madya produced Panatyaya(Madatyaya) ${ }^{7}$. it is similar to alcohol withdrawal syndrome. In Madatyaya, all the three doshas could be involved, so management should be aimed primarily at pacifying the most predominant dosha. if all the doshes are aggravated equally then, kapha should be pacified first, followed by Pitta and Vata respectively.The chronic condition are usually of Pitta and Vata aggravation and needs its management ${ }^{8}$. Case Report: This is a case of 47 years old man working as a contraction base company from Magra Punjala, Dist. Jodhpur Rajasthan came at DSRRAU, Jodhpur de-addiction unit, OPD No. 13
(Reg.No.23872) on 8/06/2019. He receives treatment under OPD after taking of history and complete physical examination. The patient was presented with nausea, vomiting, tremors, loss of appetite, constipation, disturbed sleep, slurred speech, headache. Drug History: At the age of 35 years, he started the intake of alcohol due to peer group pressure. Initially there was only occasional use, but later it developed into frequent use of alcohol. He tried to get rid of it but he fails to do so.

Personal History:

Ahara: Vegetarian; Kostha: Madhyam; Aharvidhi: Vismashan; Tea: Takes tea four times a day;

Nidra: Alapnindra, Emotional make up: Depression Astvidh Pariksha:

1) Nadi (Pulse): 78/min; 2) Mootra (urine): yellowish discolouration; 3) Mala (bowel): constipation

4) Jihwa (tongue): coated; 5) Shabda (speech): slurred speech; 6) Sparsha: Samanya; 7) Drukh: yellowish discolouration of eyes, 8) Aakruti: Madhyam

Vital Examination

Blood pressure: 130/80 mmHg

Resp. Rate: $19 / \mathrm{min}$.

Pulse Rate: $80 / \mathrm{min}$

Temperature: 98 F Height: 5 feet 11 inch

Weight: kgs:52 Kg

Liver: Enlarged Abdomen: Pain Bowel: Constipation

Ayurvedic Management of Addicted Patient of Alcohol Withdrawal Syndrome.

In OPD the prescribed medicine is advised for 15 days.

Table 1: Nashamukti yog -1(Kalpit yog)

\begin{tabular}{|l|l|l|l|l|}
\hline S.N. & Ingredient & Botanical Name & Quantity & Part Used \\
\hline 1. & Shunthi $^{9}$ & Zingiber Officinale & 1 Part & Kand \\
\hline 2. & ${\text { Choti } \text { Pippali }^{10}}^{\text {Piper Longam }}$ & 1 Part & Root \\
\hline 3. & Maricha $^{11}$ & Piper Nigrum & 1 Part & Fruit \\
\hline 4. & White Jiraka $^{12}$ & Cuminum Cyminum & 1 Part & Seed \\
\hline 5. & Ajvain $^{13}$ & Trachyspermum Ammi & 1 Part & Fruit \\
\hline 6. & Aamla $^{14}$ & Emblica Officinalis & 1 Part & Fruit \\
\hline 7. & ${\text { Seindhava } \text { Lavana }^{15}}^{\text {Seindhava Lavana }}$ & 1 Part & Salt \\
\hline 8. & Nimbbu $^{16}$ & Citrus Limon & 5 Part & Fruit Juice \\
\hline
\end{tabular}


Dose 2 Tab BD

Anupan- Water

1. Combination of Ashvagandha churna - 1gm, Shatavari churna - 1gm and Chopchini churna-1 gm, Anupaan - milk, drugs are continuous through complete treatment follow-up.

2. Madhuyashti churna ${ }^{17}-3$ gm BD

3. Shiva gutika ${ }^{18}-2$ Tab. BD, Anupan-Milk

4. Panchasakara churna ${ }^{19}-2 \mathrm{gm} \mathrm{BD}$

5. Syp. M. liv - 2 tsf BD

6. Shirobhyanga - Massage of Head by Mahanarayan taila + Til taila, Early morning for 15 days.

Patient would be given psychological counselling, normal healthy diet and medication along with medicines.

\section{$1^{\text {st }}$ follow-up}

1. Nashamukti yog -1(Kalpit yog)

2. Combination of Ashvagandha churna - 1gm, Shatavari churna - 1gm and Chopchini churna-1 gm, Anupaan - milk, drugs are continuous through complete treatment follow-up.

3. Madhuyashti Churna-3 gm BD

4. Panchasakara churna-2gm BD

5. Syp. M. liv -2 tsf BD

6. Amritkumbha -2 tsf BD

\section{$2^{\text {nd }}$ follow-up}

1. Nashamukti yog -1(Kalpit yog)

2. Combination of Ashvagandha churna - 1gm, Shatavari churna-1gm and Chopchini churna1 gm, Anupaan - milk, drugs are continuous through complete treatment follow-up.

3. Madhuyashti churna-3 gm BD

4. Panchasakara churna-2gm BD

5. Syp. M. liv -2 tsf BD

6. Amritkumbha -2 tsf BD

During the treatment and follow-ups, the patient was completely on cessation of alcohol and was advised to avoid spicy, oily, salty food and advised to take milk, Peya, and khichadi as Pathya in food

\section{Assessment Criteria}

Table 2: Clinical Institute Withdrawal Assessment of Alcohol revised scale (CIWA-Ar scale) ${ }^{20}$.

\begin{tabular}{|l|l|l|l|l|l|}
\hline S.N. & Clinical features & $\begin{array}{l}\text { Before } \\
\text { treatment } \\
\text { Score }\end{array}$ & $\begin{array}{l}\text { During } \\
\text { treatment } 15^{\text {th }} \\
\text { day }\end{array}$ & $\begin{array}{l}1^{\text {st }} \\
\text { follow- } \\
\text { up }\end{array}$ & $\begin{array}{l}2^{\text {nd }} \\
\text { follow- } \\
\text { up }\end{array}$ \\
\hline 1. & $\begin{array}{l}\text { Nausea and vomiting } \\
0=\text { no nausea and no vomiting } \\
1=\text { mild nausea with no vomiting } \\
4=\text { intermittent nausea with dry heaves } \\
7=\text { constant nausea, frequent dry heaves and vomiting }\end{array}$ & & 0 & 0 \\
\hline 2. & $\begin{array}{l}\text { Tremor } \\
0=\text { no tremor } \\
1=\text { not visible, but can be felt } \\
\text { fingertip to fingertip } \\
4=\text { moderate, with Patient's extended } \\
7=\text { severe, even with arms not extended }\end{array}$ & 4 & 1 & \\
\hline 3. & $\begin{array}{l}\text { Paroxysmal sweats } \\
0=\text { no sweat visible } \\
1=\text { barely perceptible sweating, palms moist } \\
4=\text { beads of sweat obvious on forehead } \\
7=\text { drenching sweats }\end{array}$ & & & \\
\hline 4. & $\begin{array}{l}\text { Anxiety } \\
0=\text { no anxiety } \\
1=\text { mild anxious }\end{array}$ & & & \\
\hline
\end{tabular}




\begin{tabular}{|c|c|c|c|c|c|}
\hline & $\begin{array}{l}4=\text { moderately anxious, or guarded, so anxiety is } \\
\text { inferred } \\
7=\text { equivalent to acute panic states as seen in severe } \\
\text { delirium or acute schizophrenic reactions }\end{array}$ & & & & \\
\hline 5. & $\begin{array}{l}\text { Agitation } \\
0=\text { normal activity } \\
1=\text { somewhat normal activity } \\
4=\text { moderately fidgety and restless } \\
7=\text { paces back and forth, or constantly thrash about }\end{array}$ & 1 & 0 & 0 & 0 \\
\hline 6. & $\begin{array}{l}\text { Tactile disturbance } \\
0=\text { none } \\
1=\text { very mild itching, pins \& needles, burning, or } \\
\text { numbness } \\
2=\text { mild itching, pins \& needles, burning, or numbness } \\
3=\text { moderate itching, pins \& needles, burning, or } \\
\text { numbness } \\
4=\text { moderate hallucinations } \\
5=\text { severe hallucinations } \\
6=\text { extremely severe hallucinations } \\
7=\text { continuous hallucinations }\end{array}$ & 0 & 0 & 0 & 0 \\
\hline 7. & $\begin{array}{l}\text { Visual disturbances } \\
0=\text { not present } \\
1=\text { very mild sensitivity } \\
2=\text { mild sensitivity } \\
3=\text { moderate sensitivity } \\
4=\text { moderate hallucinations } \\
5=\text { severe hallucinations } \\
6=\text { severe hallucinations } \\
7=\text { continuous hallucinations }\end{array}$ & 0 & 0 & 0 & 0 \\
\hline 8. & $\begin{array}{l}\text { Headache } \\
0=\text { not present } \\
1=\text { very mild } \\
2=\text { mild } \\
3=\text { moderate } \\
4=\text { moderately severe } \\
5=\text { severe } \\
6=\text { very severe } \\
7=\text { extremely severe }\end{array}$ & 5 & 3 & 2 & 1 \\
\hline 9. & $\begin{array}{l}\text { Auditory disturbances } \\
0=\text { not present } \\
1=\text { very mild harshness or ability to startle } \\
2=\text { mild harshness or ability to startle } \\
3=\text { moderate harshness or ability to startle } \\
4=\text { moderate hallucinations } \\
5=\text { severe hallucinations } \\
6=\text { severe hallucinations } \\
7=\text { continuous hallucinations }\end{array}$ & 0 & 0 & 0 & 0 \\
\hline 10. & Orientation and clouding of sensorium & 1 & 0 & 0 & 0 \\
\hline
\end{tabular}


$0=$ oriented

$1=$ cannot do serial addition or is uncertain about date

$2=$ disoriented to date by no more than 2 calendar days

$3=$ disoriented to date by more than 2 calendar days

$4=$ disoriented to place and /or person

Total Score

20

09

02

00

Scale for Scoring:

Table 3: Total Score

\begin{tabular}{|l|l|}
\hline Range & Withdrawal \\
\hline $0-9$ & Absent or minimal withdrawal \\
\hline $10-19$ & Mild to moderate withdrawal \\
\hline More than 20 & Severe withdrawal \\
\hline
\end{tabular}

\section{DISCUSSION}

Acharya Vagbhata explains the Madataya treatment to be done to 7 or 8 days to overcome the ill effects ${ }^{21}$, which is quite correct in case of Alcohol withdrawal syndrome. It has been explained that the symptoms due to localization of Madya in improper channels will be exhibiting only for 7 or 8 days and treatment is needed for those days. Mild to moderate symptoms on stoppage of consumption of alcohol subsides by 7 or 8 days, with treatment from our experience Nausea and vomiting, Anxiety, Tremor, Paroxysmal sweats, Headache, Agitation and clouding of sensorium are the major symptoms of alcohol withdrawal syndrome.

\section{CONCLUSION}

Addicted patient of Alcohol managed by ayurvedic principle and drugs without complication.

\section{REFERENCES}

1. Vidhyadhar Shulk, Charak Samhita Vol.2, Chikitsa Sthan 24/6, Delhi; Chaukhambha Sanskrit Sansthan; 2009. P-579

2. Prof. Krs Murthy, Susruta Samhita, Vol.3, $4^{\text {th }}$ Edi, Uttarsthan, 47/14, Varanasi; Chaukhamba Orentalia; 2017. P-307

3. Sattyanarayana Shashtri, Charaka Samhita, Vol 2, Chikitsasthan24/32, Varanasi; Chaukhamba Surbharti Prakashan; 2018 P-671

4. Sattyanarayana Shashtri, Charaka Samhita, Vol 2, Chikitsasthan 24/28, Varanasi; Chaukhamba Surbharti Prakashan; 2018 P-671
5. Dr. Ambikadatta Shastri, Susruta Samhita, Vol.2, Uttarsthan, 47/9, Varanasi; Chaukhamba Orentalia; 2017. P-419

6. Vidhyadhar Shulk, Charak Samhita Vol.2, Chikitsa Sthan 24/106, Delhi; Chaukhambha Sanskrit Sansthan; 2009. P-590

7. Pandit Hemaraja Sharma, Kasyapa Samhita, Madatyayachikitsa Adhyaya/5, Varanasi; Chaukhambha Sanskrit Sansthan; Reprint 2018. P-202

8. Vidhyadhar Shulk, Charak Samhita Vol.2, Chikitsa Sthan 24/107-108, Delhi; Chaukhambha Sanskrit Sansthan; 2009.P-590

9. Prof. K.C. Chunekar, Bhavprakashnighantu, Haritkiyaadi Varga/44-52, Varanasi; Chaukhamba Bharati Academy; 2010. P- 12

10. Prof. K.C. Chunekar, Bhavprakashnighantu, Haritkiyaadi Varga/64-65, Varanasi; Chaukhamba Bharati Academy; 2010. P- 19

11. Sattyanarayana Shashtri, Charaka Samhita, Vol 1, Sutrasthan 27/298, Varanasi; Chaukhamba Surbharti Prakashan; 2018 P-560

12. Aacharya Priyavata Sharma, Dhanvantri Nighantu, $4^{\text {th }}$ Edi, Shatpushapadi Varga/68-69, Varanasi; Chaukhamba Krishanadas Academy;2006. P-372

13. Aacharya Priyavata Sharma, Dhanvantri Nighantu, $4^{\text {th }}$ Edi, Shatpushapadi Varga/88-89, Varanasi; Chaukhamba Orentalia;2005. P-86

14. Dr. Intradev Tripathi, Rajnighantu, $4^{\text {th }}$ Edi., Aamradiphal Verga/159, Varanasi; Chaukhamba Orentalia;2006. P-372

15. Prof. K.C. Chunekar, Bhavprakashnighantu, Haritkiyaadi Varga/241, Varanasi; Chaukhamba Bharati Academy; 2010. P- 148 
16. Prof. K.C. Chunekar, Bhavprakashnighantu, Aamradiphal Verga/139-140, Varanasi; Chaukhamba Bharati Academy; 2010. P- 19

17. Prof. Siddhinandan Mishra, Bhashajaya Ratanavali, Vajikaranaadhikar 74/31, Varanasi; Chaukhamba Surbharti Prakashan;2016. P-1127

18. Prof. Siddhinandan Mishra, Bhashajaya Ratanavali, Rasayanadhikar 93/161-171, Varanasi; Chaukhamba Surbharti Prakashan;2016. P1120

19. Shree Krishanaram Gupta, Siddhabheshajmanimala, $4^{\text {th }}$ Edi. Udavart Chikitsa 4/7-8, Varanasi; Chaukhamba Krishandas Academy; 2008 P- 257

20. Sullivan, Jt Sykora Assessment Of Alcohol Withdrawal Assrss, Ent For Alcohol Scale." British Journal Of Addiction 84(11): $\quad 1353-7 \quad$ Citeseerx 10.1.489.341 Doi; $10.1111 / \mathrm{J} .1360$

21. Ram Kumar Rai, Vangsen Samhita (Chikitsasara Sangraha), $1^{\text {st }}$ Edition, Chapter-Madatyaadhiyay 20/47, Varanasi; Parchya Prakashan; 1983. P-241

\section{Source of Support: Nil}

\section{Conflict of Interest: None Declared}

How to cite this URL: Noori Aara et al: A Case Study Of Alcoholic Patient. International Ayurvedic Medical Journal \{online\} 2020 \{cited December, 2020\} Available from: http://www.iamj.in/posts/images/upload/5421 5426.pdf 